WUB 03-11

ITP-BUDAPEST 603

DESY 03-163

hep-ph/0310112

\title{
Strongly interacting neutrinos as the highest energy cosmic rays *
}

\author{
Z. Fodor ${ }^{1,2}$, S.D. Katz ${ }^{3 \star \star}$, A. Ringwald ${ }^{3}$, and H. $\mathrm{Tu}^{3}$ \\ 1 Department of Physics, University of Wuppertal, Germany \\ 2 Institute for Theoretical Physics, Eötvös University, Budapest, Hungary \\ 3 Deutsches Elektronen-Synchrotron DESY, Hamburg, Germany
}

\begin{abstract}
We show that all features of the ultrahigh energy cosmic ray spectrum from $10^{17} \mathrm{eV}$ to $10^{21} \mathrm{eV}$ can be described with a simple power-like injection spectrum of protons under the assumption that the neutrino-nucleon cross-section is significantly enhanced at center of mass energies above $\approx 100 \mathrm{TeV}$. In our scenario, the cosmogenic neutrinos produced during the propagation of protons through the cosmic microwave background initiate air showers in the atmosphere, just as the protons. The total air shower spectrum induced by protons and neutrinos shows excellent agreement with the observations. A particular possibility for a large neutrino-nucleon cross-section exists within the Standard Model through electroweak instanton-induced processes.
\end{abstract}

\section{Introduction}

The spectrum of cosmic rays extends in energy up to almost $10^{21} \mathrm{eV}$. About twenty mysterious events were observed above $10^{20} \mathrm{eV}$ by five different air shower observatories (AGASA [1], Fly's Eye [2], Haverah Park [3], HiRes [4], and Yakutsk [5]). Though some small-angle clustering in the arrival direction of the ultrahigh energy cosmic rays (UHECRs) is observed, the overall event distribution is isotropic. This indicates that they originate from several, isotropically distributed sources.

Nucleons produced at large distances with energies above the Greisen-Zatsepin-Kuzmin $(\mathrm{GZK})$ cutoff [6] $E_{\mathrm{GZK}} \approx 4 \cdot 10^{19} \mathrm{eV}$ interact with the cosmic microwave background (CMB) and produce pions which decay into neutrinos. This way the nucleons lose their energy during propagation. The typical interaction length of nucleons above $E_{\mathrm{GZK}}$ is around $50 \mathrm{Mpc}$. Thus all events above $10^{20} \mathrm{eV}$ should originate from small distances. However, no source within a distance of $50 \mathrm{Mpc}$ is known in the arrival directions of the post-GZK events. The angular distribution of UHECRs above $E_{\mathrm{GZK}}$ does not show a correlation with our galactic plane which also indicates that they originate from large distances. No conventional explanation exists to the problem how can they reach us with energies above $10^{20} \mathrm{eV}$ without an apparent energy loss.

At the relevant energies, among the known particles only neutrinos can propagate without significant energy loss from cosmological distances to us. It is this

\footnotetext{
* Talk given at Beyond the Desert '03, Castle Ringberg, 9-14 June, 2003.

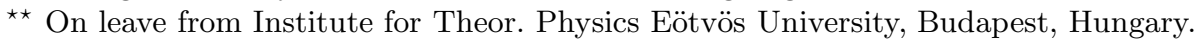


fact which led, on the one hand, to scenarios invoking hypothetical - beyond the Standard Model - strong interactions of ultrahigh energy cosmic neutrinos [7] and, on the other hand, to the Z-burst scenario [8].

Interestingly, the flux of neutrinos coming from the pions produced during the propagation of nucleons - the cosmogenic neutrinos [7] - shows a nice agreement with the observed UHECR flux above $E_{\mathrm{GZK}}[9,10]$. Assuming a large enough neutrino-nucleon cross-section at these high energies, these neutrinos could initiate extensive air showers high up in the atmosphere, like hadrons, and explain the existence of the post-GZK events. This large cross-section is usually ensured by new types of $\mathrm{TeV}$-scale interactions beyond the Standard Model, such as arising through gluonic bound state leptons [11], TeV-scale grand unification with leptoquarks [12], or Kaluza-Klein modes from compactified extra dimensions [13] (see, however, Ref. [14]); for earlier and further proposals, see Refs. [15] and [16], respectively.

In this review we discuss strongly interacting neutrino scenarios to solve the GZK problem, and in particular give an example which - in contrast to previous proposals - is based entirely on the Standard Model of particle physics. It exploits non-perturbative electroweak instanton-induced processes for the interaction of cosmogenic neutrinos with nucleons in the atmosphere, which may have a sizeable cross-section above a threshold energy $E_{\mathrm{th}}=\mathcal{O}\left(\left(4 \pi m_{W} / \alpha_{W}\right)^{2}\right) /\left(2 m_{p}\right)=$ $\mathcal{O}\left(10^{18}\right) \mathrm{eV}$, where $m_{W}$ denotes the $\mathrm{W}$-boson mass and $\alpha_{W}$ the electroweak fine structure constant [17-19]. We present a detailed statistical analysis of the agreement between observations and predictions from strongly interacting neutrino scenarios.

Our scenario is based on a standard power-like primary spectrum of protons injected from sources at cosmological distances. After propagation, these protons

will have energies below $E_{\mathrm{GZK}}$, so they can well describe the low energy part of the UHECR spectrum. The cosmogenic neutrinos interact with the atmosphere and thus give a second component to the UHECR flux, which describes the high energy part of the spectrum. The relative normalization of the proton and neutrino fluxes is fixed in this scenario, so the low and high energy parts of the spectrum are explained simultaneously without any extra normalization. Details of this analysis can be found in Ref. [20].

The structure of this review is as follows. In the next section we give the fluxes of protons and cosmogenic neutrinos both at their production and at detection. In Sect. 3 the possibility of using electroweak instantons as a source for large cross-section is discussed and the induced air shower rate is calculated. In Sect. 4 we compare the predictions with observation and determine the goodness of fit, while conclusions are given in Sect. 5 .

\section{Proton and cosmogenic neutrino fluxes}

We start with a power-like injection spectrum per co-moving volume of protons with energy $E_{i}$, spectral index $\alpha$, and redshift $(z)$ evolution index $n$,

$$
j_{p}=j_{0} E_{i}^{-\alpha}(1+z)^{n} \theta\left(E_{\max }-E_{i}\right) \theta\left(z-z_{\min }\right) \theta\left(z_{\max }-z\right) .
$$


Here, $j_{0}$ is a normalization factor, $E_{\max }$ is the maximal energy, which can be reached through astrophysical accelerating processes in a bottom-up scenario, and $z_{\min / \max }$ takes into account the absence of nearby/very early sources. The overall normalization $j_{0}$ will be fixed by the observed flux, and our predictions are quite insensitive to the specific choice for $E_{\max }, z_{\min }$, and $z_{\max }$, within their anticipated values. The main sensitivity arises from the spectral parameters $\alpha$ and $n$, for which we determine the 1 - and 2-sigma confidence regions in Sect. 4 .

The propagation of particles can be described $[9,21]$ by $P_{b \mid a}\left(r, E_{i} ; E\right)$ functions, which give the expected number of particles of type $b$ above the threshold energy $E$ if one particle of type $a$ started at a distance $r$ with energy $E_{i}$. With the help of these propagation functions, the differential flux of protons $(b=p)$ and cosmogenic neutrinos $\left(b=\nu_{i}, \bar{\nu}_{i}\right)$ at earth can be given as

$$
F_{b}(E)=\frac{1}{4 \pi} \int_{0}^{\infty} \mathrm{d} E_{i} \int_{0}^{\infty} \mathrm{d} r(-) \frac{\partial P_{b \mid p}\left(r, E_{i} ; E\right)}{\partial E} j_{p}\left(r, E_{i}\right) .
$$

In our analysis we go, according to $\mathrm{d} z=(1+z) H(z) \mathrm{d} r / c$, out to distances $R_{\max }$ corresponding to $z_{\max }=2$ (cf. Ref. [22]), while we choose $z_{\min }=0.012$ in order to take into account the fact that within $50 \mathrm{Mpc}$ there are no astrophysical sources of UHECRs. We use the expression $H^{2}(z)=H_{0}^{2}\left[\Omega_{M}(1+z)^{3}+\Omega_{\Lambda}\right]$ for the relation of the Hubble expansion rate at redshift $z$ to the present one. Uncertainties of the latter, $H_{0}=h 100 \mathrm{~km} / \mathrm{s} / \mathrm{Mpc}$, with $h=(0.71 \pm 0.07) \times 0.15$ [23], are included. $\Omega_{M}$ and $\Omega_{\Lambda}$, with $\Omega_{M}+\Omega_{\Lambda}=1$, are the present matter and vacuum energy densities in terms of the critical density. As default values we choose $\Omega_{M}=0.3$ and $\Omega_{\Lambda}=0.7$, as favored today. Our results turn out to be rather insensitive to the precise values of the cosmological parameters.

We calculated $P_{b \mid a}\left(r, E_{i} ; E\right)$ in two steps. $\left.i\right)$ First, the SOPHIA Monte-Carlo program [24] was used for the simulation of photohadronic processes of protons with the CMB photons. For $e^{+} e^{-}$pair production we used the continuous energy loss approximation, since the inelasticity is very small $\left(\approx 10^{-3}\right)$. We calculated the $P_{b \mid a}$ functions for "infinitesimal" steps $(1-10 \mathrm{kpc})$ as a function of the redshift $z$. ii) We multiplied the corresponding infinitesimal probabilities starting at a distance $r(z)$ down to earth with $z=0$.

Since the propagation functions are of universal usage, we decided to make the latest versions of $-\partial P_{b \mid a} / \partial E$ available for the public via the World-WideWeb URL www.desy.de/ uhecr .

\section{$3 \quad$ Neutrino induced air shower rate}

The main assumption of our scenario is that the neutrino-nucleon cross-section $\sigma_{\nu N}^{\text {tot }}$ suddenly becomes much larger than $\approx 1 \mathrm{mb}$ above center of mass energies $\sqrt{s} \approx 100 \mathrm{TeV}$. In this case, the corresponding neutrino interaction length $\lambda_{\nu} \equiv$ $m_{p} / \sigma_{\nu N}^{\text {tot }}$, with $\sigma_{\nu N}^{\text {tot }}=\sigma_{\nu N}^{\text {cc }}+\sigma_{\nu N}^{\mathrm{s}}$, falls below $X_{0}=1031 \mathrm{~g} / \mathrm{cm}^{2}-$ the vertical depth of the atmosphere at sea level - above the threshold energy $\approx 10^{19} \mathrm{eV}$. Here $\sigma_{\nu N}^{\mathrm{cc}}$ and $\sigma_{\nu N}^{\mathrm{s}}$ denote the charged current and the new contribution to the cross-section. Above the neutrino threshold energy, the atmosphere becomes 
opaque to cosmogenic neutrinos and most of them will end up as air showers. Quantitatively, this fact can be described by

$$
F_{\nu}^{\prime}(E)=F_{\nu}(E)\left[1-\mathrm{e}^{-\frac{X(\theta)}{\lambda_{\nu}(E)}}\right]
$$

which gives the spectrum of neutrino-initiated air showers, for an incident cosmogenic neutrino flux $F_{\nu}=\sum_{i}\left[F_{\nu_{i}}+F_{\bar{\nu}_{i}}\right]$ from Eq. (2), in terms of the atmospheric depth $X(\theta)$, with $\theta$ being the zenith angle.
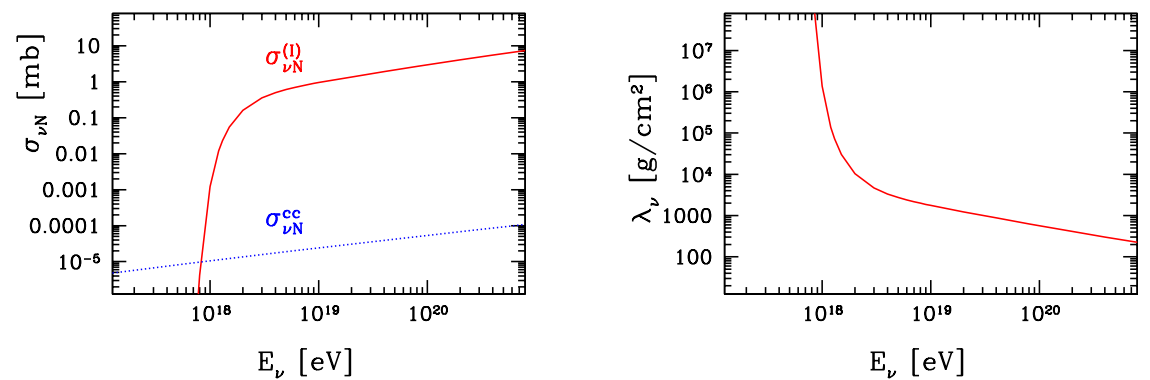

Fig. 1. Left: Prediction of the electroweak instanton-induced neutrino-nucleon crosssection $\sigma_{\nu N}^{(I)}$ (solid) in comparison with the charged current cross-section $\sigma_{\nu N}^{\mathrm{cc}}$ (dotted) from Ref. [25], as a function of the neutrino energy $E_{\nu}$ in the nucleon's rest frame. Right: Neutrino interaction length due to combined effects of charged current interactions and instanton-induced processes.

Such suddenly increasing cross-sections have been proposed in various models involving physics beyond the Standard Model [11-16]. In Fig. 1 we show another example which is based entirely on the Standard Model exploiting nonperturbative electroweak instanton-induced processes [17-19]. Our quantitative analysis [20] was based on this cross-section, however it is expected to be insensitive to the exact form of it as long as it rises abruptly far above $1 \mathrm{mb}$. Note that such a behaviour is consistent with present upper bounds on electroweak instanton-induced cross-sections [26]. It should be noted that such a cross-section will lead, via dispersion relations, to lower energy deviations of Standard Model predictions for elastic scattering from their perturbative values. However, it is easily checked that, for the one shown in Fig. 1 (left), these corrections will be unobservably small in the energy regime available at present accelerators [27].

\section{Comparison with UHECR data}

The predicted air shower rate induced by protons and neutrinos is given by

$$
F_{\text {pred }}\left(E ; \alpha, n, E_{\max }, z_{\min }, z_{\max }, j_{0}\right)=F_{p}(E ; \ldots)+F_{\nu}^{\prime}(E ; \ldots) .
$$

We performed a statistical analysis to compare (4) with the observations and presented a measure for the goodness of the scenario [20]. We gave the best fit to the observations and the 1- and 2-sigma confidence regions in the $(\alpha, n)$ plane. 
UHECR collaborations usually publish their results for the detected fluxes in a binned form. The first step of our analysis is to convert these fluxes into event numbers in each bin. We use the most recent results of the HiRes and AGASA collaborations and do our analysis separately with both data sets. We use the energy range $10^{17.2} \mathrm{eV}-10^{21} \mathrm{eV}$ which is divided into 38 equal logarithmic bins. In the low energy region, there are no published results available from AGASA and only low statistics results from HiRes-2. Therefore, we included the results of the predecessor collaborations - Akeno [28] and Fly's Eye, respectively - into the analysis.

The goodness of the scenario is determined by a statistical analysis. We determined the compatibility of different $(\alpha, n)$ pairs with the experimental data. For some fixed $(\alpha, n)$ pair, the expected number of events in individual bins are $\left(\lambda=\left\{\lambda_{1}, \ldots, \lambda_{r}\right\}\right.$ with $r$ being the total number of bins (in our case 38 ). The probability of getting an experimental outcome $\mathbf{k}=\left\{k_{1}, \ldots k_{r}\right\}$ (where $k_{i}$ are non-negative integer numbers) is given by the probability distribution function $P(\mathbf{k})$, which is just the product of Poisson distributions for the individual bins. It is easy to include also the $\approx 30 \%$ overall energy uncertainty into the $P(\mathbf{k})$ probability distribution. We denote the experimental result by $\mathbf{s}=\left\{s_{1}, \ldots s_{r}\right\}$, where the $s_{i}$-s are non-negative, integer numbers. The $(\alpha, n)$ pair is compatible with the experimental results if

$$
\sum_{\mathbf{k} \mid P(\mathbf{k})>P(\mathbf{s})} P(\mathbf{k})<c .
$$

For a 1-(or 2-)sigma compatibility one takes $\mathrm{c}=0.68$ (or $\mathrm{c}=0.95$ ), respectively. The best fit is found by minimizing the sum on the left hand side.

Since we have 38 variables, it is practically impossible to calculate the sum in equation (5) exactly. If we rewrite it as

$$
\sum_{\mathbf{k} \mid P(\mathbf{k})>P(\mathbf{s})} P(\mathbf{k})=\frac{\sum_{\mathbf{k}} P(\mathbf{k}) \theta[P(\mathbf{k})-P(\mathbf{s})]}{\sum_{\mathbf{k}} P(\mathbf{k})},
$$

then we can calculate this sum approximately using an importance sampling based Monte-Carlo technique. We have to generate the components of $\mathbf{k}$ with Poisson distribution and take only those in the sum, for which $P(\mathbf{k})>P(\mathbf{s})$.

Figure 2 (left) shows our best fits for the AGASA and for the HiRes UHECR data. The best fit values are $\alpha=2.68(2.68)$ and $n=2.65(2.9)$, for AGASA(HiRes). We can see very nice agreement with the data within an energy range of nearly four orders of magnitude. The fits are insensitive to the value of $E_{\max }$ as far as we choose a value above $\approx 3 \cdot 10^{21} \mathrm{eV}$. The shape of the curve between $10^{17} \mathrm{eV}$ and $10^{19} \mathrm{eV}$ is mainly determined by the redshift evolution index $n$. At these energies the universe is already transparent for protons created at $z \approx 0$ while protons from sources with larger redshift accumulate in this region. The peak around $4 \cdot 10^{19} \mathrm{eV}$ shows the accumulation of particles due to the GZK effect. Neutrinos start to dominate over protons at around $10^{20} \mathrm{eV}$.

It is important to note that, if we omit the neutrino component, then the model is ruled out on the 3-sigma level for both experiments. This is due to the 

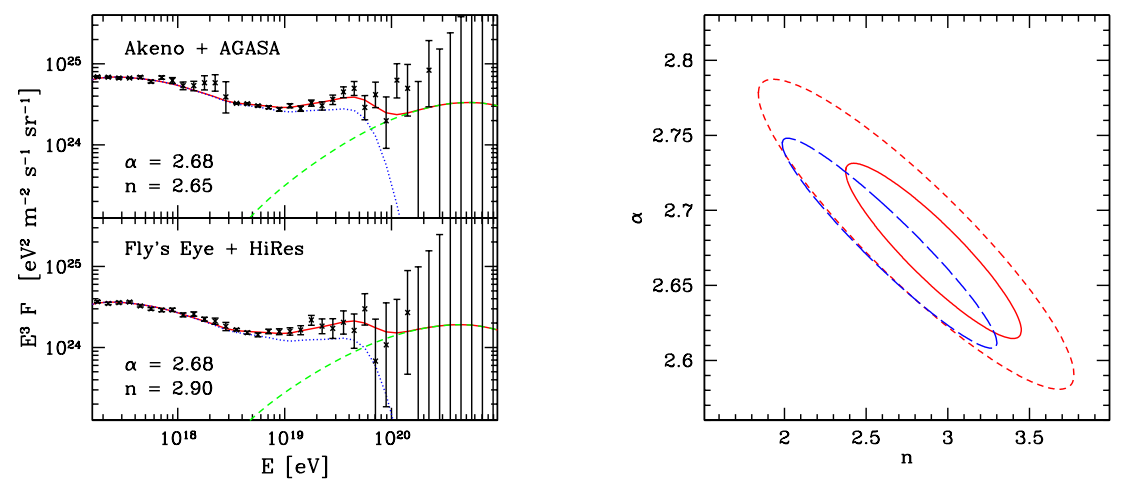

Fig. 2. Left: Ultrahigh energy cosmic ray data (Akeno + AGASA on the upper panel and Fly's Eye + HiRes on the lower panel) and their best fits within the electroweak instanton scenario (solid) for $E_{\max }=3 \cdot 10^{22} \mathrm{eV}, z_{\min }=0.012, z_{\max }=2$ consisting of a proton component (dotted) plus a cosmogenic neutrino-initiated component (dashed). Right: Confidence regions in the $\alpha-n$ plane for fits to the Akeno + AGASA data (2sigma (long dashed)) and to the Fly's Eye + HiRes data (1-sigma (solid); 2-sigma (short-dashed)), respectively.

fact that we excluded nearby sources by setting $z_{\min } \neq 0$ (see also Ref. [29]). A choice of $z_{\min }=0$ makes the HiRes data compatible with a proton-only scenario on the 2-sigma level (see also Refs. [4,30]).

Figure 2 (right) displays the confidence regions in the $(\alpha, n)$ plane for AGASA and HiRes. The scenario is consistent on the 2-sigma level with both experiments. For HiRes, the compatibility is even true on the 1-sigma level. It is important to note that both experiments favor the same values for $\alpha$ and $n$, demonstrating their mutual compatibility on the 2-sigma level (see also Ref. [31]).

Finally, let us discuss the consistency of our scenario with the currently available limits on deeply penetrating showers from Fly's Eye [32] and AGASA [33]. Taking into account - in distinction to Ref. [34] - the atmospheric attenuation of the cosmogenic neutrino flux predicted in our scenario and the uncertainties in the estimate of the range of depth within which the shower must originate to trigger the array, we find that AGASA should have seen 1 - 10 quasi-horizontal air showers $\left(\theta \gtrsim 60^{\circ}\right)$ from the electroweak instanton-induced processes during a running time of 1710.5 days. This is consistent with AGASA's present analysis of their respective data [33]. The Fly's Eye upper limit on the product of the total neutrino flux times neutrino-nucleon cross-section, $\left(F_{\nu} \sigma_{\nu N}^{\text {tot }}\right)_{\text {Fly's Eye }}[32]$, in the energy range $10^{17-20} \mathrm{eV}$, can be translated, for a given predicted neutrino flux $F_{\nu}^{\text {pred }}$, into an upper limit on $\sigma_{\nu N}^{\text {tot }}<\left(F_{\nu} \sigma_{\nu N}^{\text {tot }}\right)_{\text {Fly's Eye }} / F_{\nu}^{\text {pred }}$, as long as it is smaller than $10 \mu \mathrm{b}[18,35]$. We find that, for our predicted cosmogenic neutrino flux, the right-hand-side of this inequality is larger than $10 \mu \mathrm{b}$ in the whole energy range, such that the Fly's Eye non-observation of quasi-horizontal air showers does not give any constraint. We therefore conclude that our prediction of the neutrino-nucleon cross-section, as shown in Fig. 1 (left), does not contra- 
dict any constraints from cosmic ray experiments so far, as long as the ultrahigh energy cosmic neutrino flux is at the cosmogenic level we have predicted.

\section{$5 \quad$ Summary and conclusions}

We have shown that a simple scenario with a single power-like injection spectrum of protons can describe all the features of the UHECR spectrum in the energy range $10^{17-21} \mathrm{eV}$. In our scenario, the injected protons produce neutrinos during their propagation and these neutrinos are assumed to have large enough cross-section to produce air showers high up in the atmosphere. As an example we discussed the possibility that Standard Model electroweak instanton-induced processes may give a cross section which is suitable for this scenario. The model has few parameters from which only two - the power index $\alpha$ and the redshift evolution index $n$ - has a strong effect on the final shape of the spectrum. We found that for certain values of $\alpha$ and $n$ this scenario is compatible with the available observational data from the AGASA and HiRes experiments (combined with their predecessor experiments, Fly's Eye and Akeno, respectively) on the 2-sigma level (also 1-sigma for HiRes). The ultrahigh energy neutrino component can be experimentally tested by studying the zenith angle dependence of the events in the range $10^{18-20} \mathrm{eV}$ and possible correlations with distant astrophysical sources [36] at cosmic ray facilities such as the Pierre Auger Observatory [37], and by looking for enhanced rates for throughgoing muons at neutrino telescopes such as AMANDA [38]. As laboratory tests, one may search for a model-independent enhancement in (quasi-)elastic lepton-nucleon scattering [27] or for signatures of QCD instanton-induced processes in deep-inelastic scattering [39], e.g. at HERA.

\section{Acknowledgments}

This work was partially supported by Hungarian Science Foundation grants No. OTKA-T034980/T037615.

\section{References}

1. M. Takeda et al., Phys. Rev. Lett. 81 (1998) 1163;

2. D. J. Bird et al., Phys. Rev. Lett. 71 (1993) 3401; Astrophys. J. 424 (1994) 491; ibid. 441 (1995) 144.

3. M. A. Lawrence, R. J. Reid, A. A. Watson, J. Phys. G 17 (1991) 733; M. Ave, et al., Phys. Rev. Lett. 85 (2000) 2244.

4. T. Abu-Zayyad et al. [HiRes Collaboration], astro-ph/0208243; astro-ph/0208301.

5. N.N. Efimov et al., in Proc. of the Astrophysical Aspects of the Most Energetic Cosmic Rays (World Scientific, Singapore, 1991).

6. K. Greisen, Phys. Rev. Lett. 16 (1966) 748; G. T. Zatsepin and V. A. Kuzmin, JETP Lett. 4 (1966) 78 [Pisma Zh. Eksp. Teor. Fiz. 4 (1966) 114].

7. V. S. Beresinsky and G. T. Zatsepin, Phys. Lett. B 28 (1969) 423. 
8. D. Fargion et al., Astrophys. J. 517 (1999) 725; T. J. Weiler, Astropart. Phys. 11 (1999) 303; S. Yoshida, G. Sigl and S. J. Lee, Phys. Rev. Lett. 81 (1998) 5505; Z. Fodor, S. D. Katz and A. Ringwald, Phys. Rev. Lett. 88 (2002) 171101; JHEP 0206 (2002) 046; O. E. Kalashev et al., Phys. Rev. D 65 (2002) 103003.

9. S. Yoshida and M. Teshima, Prog. Theor. Phys. 89 (1993) 833.

10. R. J. Protheroe and P. A. Johnson, Astropart. Phys. 4 (1996) 253; S. Yoshida et al., Astrophys. J. 479 (1997) 547; R. Engel and T. Stanev, Phys. Rev. D 64 (2001) 093010; O. E. Kalashev et al., Phys. Rev. D 66 (2002) 063004; Z. Fodor, S. D. Katz, A. Ringwald and H. Tu, hep-ph/0309171; D. Semikoz and G. Sigl, hep-ph/0309328.

11. J. Bordes et al., hep-ph/9705463; Astropart. Phys. 8 (1998) 135.

12. G. Domokos, S. Kovesi-Domokos and P. T. Mikulski, hep-ph/0006328.

13. G. Domokos and S. Kovesi-Domokos, Phys. Rev. Lett. 82 (1999) 1366; S. Nussinov and R. Shrock, Phys. Rev. D 59 (1999) 105002; P. Jain et al., Phys. Lett. B 484 (2000) 267.

14. M. Kachelriess and M. Plümacher, Phys. Rev. D 62 (2000) 103006; L. Anchordoqui et al., Phys. Rev. D 63 (2001) 124009.

15. G. Domokos and S. Nussinov, Phys. Lett. B 187 (1987) 372.

16. S. Barshay and G. Kreyerhoff, Eur. Phys. J. C 23 (2002) 191; Phys. Lett. B 535 (2002) 201; L. A. Anchordoqui, J. L. Feng and H. Goldberg, Phys. Lett. B 535 (2002) 302.

17. H. Aoyama and H. Goldberg, Phys. Lett. B 188 (1987) 506; A. Ringwald, Nucl. Phys. B 330 (1990) 1; O. Espinosa, Nucl. Phys. B 343 (1990) 310; V. V. Khoze and A. Ringwald, Phys. Lett. B 259 (1991) 106.

18. D. A. Morris and A. Ringwald, Astropart. Phys. 2 (1994) 43.

19. A. Ringwald, Phys. Lett. B 555 (2003) 227.

20. Z. Fodor, S. D. Katz, A. Ringwald and H. Tu, Phys. Lett. B 561 (2003) 191.

21. J. N. Bahcall and E. Waxman, Astrophys. J. 542 (2000) 543; Z. Fodor and S. D. Katz, Phys. Rev. D 63 (2001) 023002.

22. E. Waxman, Astrophys. J. 452 (1995) L1.

23. K. Hagiwara et al. [Particle Data Group], Phys. Rev. D 66 (2002) 010001.

24. A. Mücke et al., Comput. Phys. Commun. 124 (2000) 290.

25. R. Gandhi, C. Quigg, M. H. Reno and I. Sarcevic, Phys. Rev. D 58 (1998) 093009.

26. F. Bezrukov et al., Phys. Rev. D 68 (2003) 036005; A. Ringwald, hep-ph/0307034; T. Han and D. Hooper, hep-ph/0307120.

27. H. Goldberg and T. J. Weiler, Phys. Rev. D 59 (1999) 113005.

28. M. Nagano et al., J. Phys. G 18 (1992) 423.

29. M. Kachelriess, D. V. Semikoz and M. A. Tortola, Phys. Rev. D 68 (2003) 043005.

30. J. N. Bahcall and E. Waxman, Phys. Lett. B 556 (2003) 1.

31. D. De Marco, P. Blasi and A. V. Olinto, Astropart. Phys. 20 (2003) 53.

32. R. M. Baltrusaitis et al., Phys. Rev. D 31 (1985) 2192.

33. S. Yoshida et al. [AGASA Collaboration], in Proc. ${ }^{27}$ th International Cosmic Ray Conference, Hamburg, Germany, 2001, Vol. 3, p. 1142.

34. L. A. Anchordoqui et al., Phys. Rev. D 66 (2002) 103002.

35. C. Tyler, A. V. Olinto and G. Sigl, Phys. Rev. D 63 (2001) 055001; A. Ringwald and H. Tu, Phys. Lett. B 525 (2002) 135.

36. P. G. Tinyakov and I. I. Tkachev, JETP Lett. 74 (2001) 445 [Pisma Zh. Eksp. Teor. Fiz. 74 (2001) 499]; Astropart. Phys. 18 (2002) 165.

37. D. Zavrtanik [AUGER Collaboration], Nucl. Phys. Proc. Suppl. 85 (2000) 324.

38. E. Andres et al. [AMANDA Collaboration], Nature 410 (2001) 441.

39. A. Ringwald and F. Schrempp, hep-ph/9411217; Phys. Lett. B 503 (2001) 331. 